\title{
Push-pull optimization of quantum controls
}

\author{
Priya Batra, ${ }^{*}$ V. R. Krithika, ${ }^{\dagger}$ and T. S. Mahesh ${ }^{\ddagger}$ \\ Department of Physics and NMR Research Center, Indian Institute of Science Education and Research, Pune 411008, India
}

(Received 26 September 2019; accepted 26 January 2020; published 13 March 2020)

\begin{abstract}
Optimization of quantum controls to achieve a target process is centered around an objective function comparing the realized process with the target. We propose an objective function that incorporates not only the target operator but also a set of its orthogonal operators whose combined influence leads to an efficient exploration of the parameter space, faster convergence, and extraction of superior solutions. The push-pull optimization, as we call it, can be adopted in various quantum control scenarios. We describe adopting it for gradient based and variational-principle based approaches. Numerical analysis of quantum registers with up to seven qubits reveals significant benefits of the push-pull optimization. We describe applying the push-pull optimization to prepare a long-lived singlet order in a two-qubit system using NMR techniques.
\end{abstract}

DOI: 10.1103/PhysRevResearch.2.013314

\section{INTRODUCTION}

Optimal control theory finds applications in diverse fields such as finance, science, and engineering [1,2]. Quantum optimal control has also gained significant attention over the past several years [3,4] and is routinely used in robust steering of quantum dynamics such as in chemical kinetics [5,6], spectroscopy [7-9], quantum computing [10,11], and many more [12]. Here we focus on optimization of quantum controls either to transfer from one state to another, henceforth called state control, or to realize a target unitary evolution, henceforth called gate control. Relevant numerical techniques fall into several categories including stochastic-search methods such as strongly modulating pulses [13], gradient-based approaches such as gradient ascent pulse engineering (GRAPE) [14,15] and gradient optimization of analytical control [16], variational-principle-based Krotov optimization [17-19], truncated basis approaches such as chopped random basis optimization [20,21], genetic algorithm enabled bang-bang controls [22,23], and machine-learningbased approaches $[24,25]$. These control schemes have been implemented on various quantum architectures such as NMR [9,13,22,26], nitrogen-vacancy centers [27], ion traps [28], superconducting qubits [29], magnetic resonance imaging [30], and cold atoms [11].

An objective function evaluating the overlap of the realized process with the target process is at the core of an optimization algorithm and therefore should be chosen carefully $[31,32]$. Here we propose a hybrid objective function that

\footnotetext{
*priya.batra@students.iiserpune.ac.in

${ }^{\dagger}$ krithika_vr@students.iiserpune.ac.in

${ }^{\ddagger}$ mahesh.ts@iiserpune.ac.in
}

Published by the American Physical Society under the terms of the Creative Commons Attribution 4.0 International license. Further distribution of this work must maintain attribution to the author(s) and the published article's title, journal citation, and DOI. depends not only on the target operator, but also on a set of orthogonal operators. One may think of control parameters as being pulled by the target operator as well as pushed by the orthogonal operators. Accordingly, we refer to this method as push-pull optimization of quantum controls (PPOQC). We describe adopting PPOQC for GRAPE and Krotov algorithms and demonstrate its superior convergence over the standard pull-only methods. We also experimentally demonstrate the efficacy of PPOQC in a NMR quantum test bed by preparing long-lived singlet order.

The article is organized as follows. In Sec. II we describe the optimization problem and the push-pull approach, particularly focusing on a gradient-based algorithm as well as a variational-principle-based algorithm. In Sec. III we provide numerical analysis to compare push-pull with pull-only approaches. Here we also study the dependence of the push-pull approach on various aspects such as system size, conjugate gradients, and adaptive weights. In Sec. IV we describe the NMR experiments to generate the long-lived singlet order in a two-spin system directly from thermal equilibrium using a pulse sequence designed by the push-pull Krotov algorithm. We summarize in Sec. V.

\section{OPTIMIZATION PROBLEM}

Consider a quantum system with an internal or fixed Hamiltonian $H_{0}$ and a set of $M$ control operators $\left\{A_{k}\right\}$ leading to the full time-dependent Hamiltonian $H(t)=H_{0}+$ $\sum_{k=1}^{M} u_{k}(t) A_{k}$, where control amplitudes $u_{k}(t)$ are amenable to optimization. The propagator for a control sequence of duration $T$ is $D \exp \left[-i \int_{0}^{T} H\left(t^{\prime}\right) d t^{\prime}\right]$, where $D$ is the Dyson timeordering operator. The standard approach to simplifying the propagator is via piecewise-constant control amplitudes with $N$ segments each of duration $\tau$ [see Fig. 1(a)]. In this case, the overall propagator is of the form $U_{1: N}=U_{N} U_{N-1} \cdots U_{2} U_{1}$, where $U_{j}=\exp \left(-i H_{j} \tau\right)$ is the propagator for the $j$ th segment and $H_{j}=H_{0}+\sum_{k=1}^{M} u_{j k} A_{k}$. Our task is to optimize the control sequence $\left\{u_{j k}\right\}$ depending on the following two kinds of optimizations. 

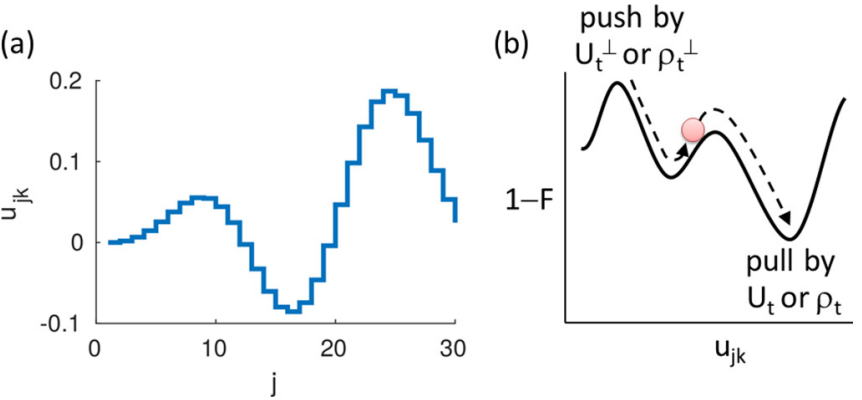

FIG. 1. (a) Piecewise-constant control parameter $u_{j k}$ versus the segment number $j$. (b) Infidelity $1-F$ versus $u_{j k}$.

(i) Gate control (GC). Here the goal is to achieve an overall propagator (gate) $U_{t}$ that is independent of the initial state. This is realized by maximizing the gate fidelity $F\left(U_{1: N}, U_{t}\right)=$ $\left|\left\langle U_{t} \mid U_{1: N}\right\rangle\right|^{2}=\left|\operatorname{Tr}\left\{U_{t}^{\dagger} U_{1: N}\right\}\right|^{2}$.

(ii) State control $(S C)$. Here the goal is to drive a given initial state $\rho_{0}$ to a desired target state $\rho_{t}$. This can be achieved by maximizing the state fidelity $F\left(\rho_{1: N}, \rho_{t}\right)=\left\langle\rho_{t} \mid \rho_{1: N}\right\rangle=$ $\operatorname{Tr}\left\{\rho_{t} \rho_{1: N}\right\}$, where $\rho_{1: N}=U_{1: N} \rho_{0} U_{1: N}^{\dagger}$.

In practice, hardware limitations impose bounds on the control parameters $\left\{u_{j k}\right\}$ and therefore it is desirable to minimize the overall control resource $r_{k}=\sum_{j} u_{j k}^{2}$. To this end, we use the performance function $J=F-\sum_{k=1}^{M} \lambda_{k} r_{k}$, where $\lambda_{k}$ are penalty constants.

$P P O Q C$. Be it gate control or state control, for a $d$ dimensional target operator, we can efficiently setup $d-1$ orthogonal operators via the Gram-Schmidt orthogonalization procedure [33]. The target operator pulls the control sequence towards itself, whereas the orthogonal operators push it away from them [see Fig. 1(b)]. We define the push fidelities as

$$
\begin{aligned}
& F_{o}\left(U_{1: N},\left\{V_{l}\right\}\right)=\frac{1}{L} \sum_{l=1}^{L} F\left(U_{1: N}, V_{l}\right) \text { for } \mathrm{GC}, \\
& F_{o}\left(\rho_{1: N},\left\{R_{l}\right\}\right)=\frac{1}{L} \sum_{l=1}^{L} F\left(\rho_{1: N}, R_{l}\right) \text { for } \mathrm{SC},
\end{aligned}
$$

where $\left\{V_{l}\right\}$ and $\left\{R_{l}\right\}$ are $L \leqslant d-1$ orthogonal operators such that $F\left(U_{t}, V_{l}\right)=0$ and $F\left(\rho_{t}, R_{l}\right)=0$. Of course, $d$ increases exponentially with the system size, but as we will see later, a small subset of $L$ orthogonal operators can bring about a substantial advantage. Also, note that for a given target operator, the set of orthogonal operators is not unique and can be generated randomly and efficiently in every iteration. We define the push-pull performance function

$$
J_{\mathrm{PP}}=F-\alpha F_{o}-\sum_{k=1}^{M} \lambda_{k} r_{k} .
$$

The push weight $\alpha$ can be a constant or adaptively adjusted. In the following, we describe incorporating PPOQC into two popular optimal quantum control methods.

\section{A. GRAPE optimization}

Being a gradient-based approach, GRAPE optimization involves an efficient calculation of the maximum-ascent direction [14]. While it is sensitive to the initial guess and looks for a local optimum, it is nevertheless simple, powerful, and popular. The algorithm iteratively updates control parameters $\left\{u_{j k}\right\}$ in the direction of gradient $g_{j k}^{(i)}=\partial J^{(i)} / \partial u_{j k}^{(i)}$,

$$
\begin{aligned}
& g_{j k}^{(i)}\left(U_{t}\right)=2 \tau \operatorname{Im}\left\{\left\langle P_{j} \mid A_{k} U_{1: j}\right\rangle\left\langle U_{1: j} \mid P_{j}\right\rangle\right\} \text { for } \mathrm{GC}, \\
& g_{j k}^{(i)}\left(\rho_{t}\right)=-i \tau\left\langle\tilde{\rho}_{j} \mid\left[A_{k}, \rho_{1: j}\right]\right\rangle \text { for } \mathrm{SC},
\end{aligned}
$$

where $i$ denotes the iteration number, $P_{j}=U_{j+1: N}^{\dagger} U_{t}$, and $\tilde{\rho}_{j}=U_{j+1: N}^{\dagger} \rho_{t} U_{j+1: N}$ [14]. Collective updates $u_{j k}^{(i+1)}=u_{j k}^{(i)}+$ $\epsilon g_{j k}^{(i)}$ after iteration $i$ on all the segments with a suitable step size $\epsilon$ proceed with monotonic convergence.

Push-pull GRAPE (PP-GRAPE). Using Eq. (2), we recast the gradients as

$$
\begin{aligned}
& G_{j k}^{(i)}\left(U_{t},\left\{V_{l}\right\}\right)=g_{j k}^{(i)}\left(U_{t}\right)-\frac{\alpha}{L} \sum_{l=1}^{L} g_{j k}^{(i)}\left(V_{l}\right) \text { for } \mathrm{GC}, \\
& G_{j k}^{(i)}\left(\rho_{t},\left\{R_{l}\right\}\right)=g_{j k}^{(i)}\left(\rho_{t}\right)-\frac{\alpha}{L} \sum_{l=1}^{L} g_{j k}^{(i)}\left(R_{l}\right) \text { for } \mathrm{SC}
\end{aligned}
$$

and the update rule as $u_{j k}^{(i+1)}=u_{j k}^{(i)}+\epsilon G_{j k}^{(i)}$. The revised gradients form the basis of PP-GRAPE.

\section{B. Krotov optimization}

Based on the variational principle, this method aims for the global optimum [34]. Here the performance function is maximized with the help of an appropriate Lagrange multiplier $B_{j}$. One sets up a Lagrangian of the form [9]

$$
\mathcal{L}=F-\sum_{k=1}^{M} \lambda_{k} r_{k}-\sum_{j=1}^{N} \operatorname{Re}\left\langle B_{j}\left|\frac{d}{d t}+i H_{j}\right| U_{0: j}\right\rangle,
$$

where the first two terms are the same as the performance function $J$, and looks for a stationary point satisfying $\frac{\partial \mathcal{L}}{\partial F}=0$, $\frac{\partial \mathcal{L}}{\partial u_{j k}}=0$, and $\frac{\partial \mathcal{L}}{\partial B_{j}}=0$. The second differential equation leads to $u_{j k}=\frac{1}{\lambda_{k}} \operatorname{Im}\left\langle B_{j} \mid A_{k} U_{0: j}\right\rangle$ and the last differential equation constrains evolution according to the Schrödinger equation $\dot{B}(t)=-i H(t) B(t)$.

At every iteration $i$, the Krotov algorithm evaluates the control sequence $\left\{u_{j k}^{(i)}\right\}$ as well as its cosequence $\left\{\tilde{u}_{j k}^{(i)}\right\}$. Starting with a random guess $\left\{u_{j k}^{(0)}\right\}=\left\{\tilde{u}_{j k}^{(0)}\right\}$, forward propagation of the sequence $\left\{u_{j k}^{(0)}\right\}$ gives $U_{1: j}$ and backward propagation of the cosequence $\left\{\tilde{u}_{j k}^{(0)}\right\}$ from the boundary $B_{N}=\partial F / \partial U_{1: N}$ leads to $B_{j}$. Specifically,

$$
\begin{aligned}
& B_{N}=\left\langle U_{t} \mid U_{0: N}\right\rangle U_{t} \text { for } \mathrm{GC}, \\
& B_{N}=\rho_{t} U_{0: N} \rho_{0}+\kappa U_{0: N} \quad \text { for } \mathrm{SC} .
\end{aligned}
$$

Here $U_{0: N}=U_{0} U_{1: N}, U_{0}=\mathbb{1}$, and $\kappa$ is a positive constant that ensures the positivity of fidelity. Backpropagating the cosequence, we obtain

$$
B_{j}=\tilde{U}_{j+1}^{\dagger} \cdots \tilde{U}_{N-1}^{\dagger} \tilde{U}_{N}^{\dagger} B_{N},
$$



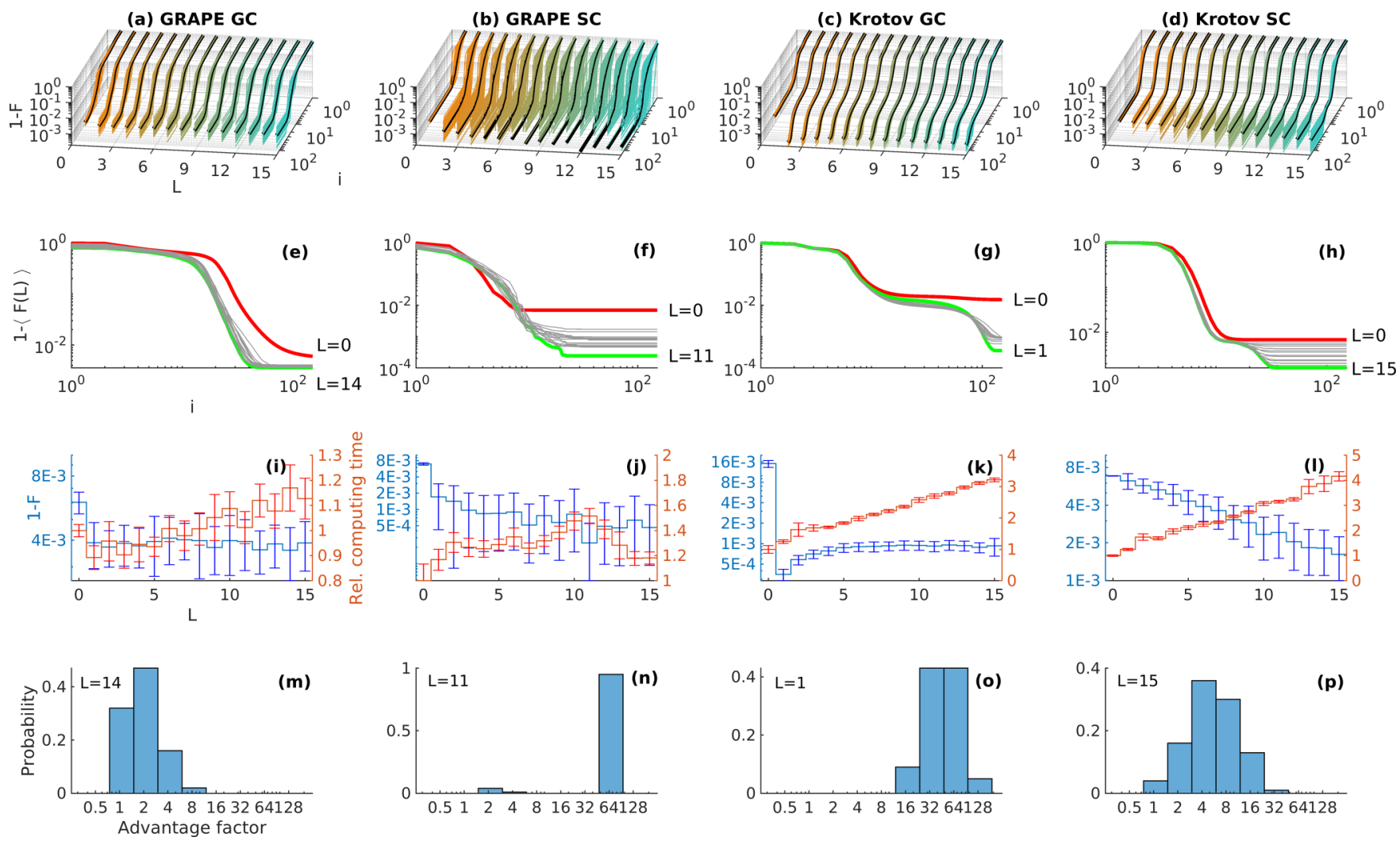

FIG. 2. Performance analysis on a two-qubit system for GRAPE GC (first column), GRAPE SC (second column), Krotov GC (third column), and Krotov SC (fourth column). (a)-(d) Infidelity $1-F$ of two-qubit controls versus iteration number $(i)$ and number $(L)$ of orthogonal operators for GRAPE and Krotov as indicated. Black lines represent mean infidelities. (e)-(h) Mean infidelity versus $i$. Curves for $L=0$ (red) and for $L$ leading to the maximum final fidelity (green) are highlighted. (i)-(l) Mean final infidelity (left axis) and relative computing time (right axis) versus $L$. Error bars represent one standard deviation. (m)-(p) Probability versus advantage factor.

where $\tilde{U}_{j}=\exp (-i \tilde{H} \tau)$ and $\tilde{H}_{j}=H_{0}+\sum_{k=1}^{M} \tilde{u}_{j k} A_{k}$. Now the sequence $\left\{u_{j k}^{(i)}\right\}$ is updated according to

$$
u_{j k}^{(i)}=(1-\delta) \tilde{u}_{j k}^{(i-1)}+\frac{\delta}{\lambda_{k}} \operatorname{Im}\left\langle B_{j}^{(i-1)} \mid A_{k} U_{0: j-1}^{(i)}\right\rangle
$$

and the propagator $U_{0: j}^{(i)}$ is evaluated. Iterating the last two steps delivers propagators $U_{0: 1}^{(i)}, U_{0: 2}^{(i)}, \ldots, U_{0: N}^{(i)}$. The terminal Lagrange multiplier $B_{N}^{(i)}$ is evaluated using Eq. (6). To set up the cosequence $\left\{\tilde{u}_{j k}^{(i)}\right\}$ we first evaluate the terminal control $\tilde{u}_{N k}$ using

$$
\tilde{u}_{j k}^{(i)}=(1-\eta) u_{j k}^{(i)}+\frac{\eta}{\lambda_{k}} \operatorname{Im}\left\langle B_{j}^{(i)} \mid A_{k} U_{0: j}^{(i)}\right\rangle,
$$

with $j=N$. The Lagrange multiplier $B_{N-1}^{(i)}=\tilde{U}_{N}^{\dagger} B_{N}^{(i)}$ is now evaluated by backpropagating with the updated amplitude $\tilde{u}_{N k}^{(i)}$. Iterating the last two steps updates the whole cosequence $\left\{\tilde{u}_{j k}^{(i)}\right\}$. The algorithm is continued until the desired fidelity is reached.

Push-pull Krotov (PP-Krotov). Here we use $L$ additional cosequences $\left\{\tilde{v}_{j k l}^{(i)}\right\}$ corresponding to orthogonal operators $\left\{V_{l}\right\}$ or $\left\{R_{l}\right\}$. Terminal Lagrange multipliers $\left\{C_{N l}\right\}$ are obtained using equations similar to Eq. (6): $C_{N l}=\left\langle V_{l} \mid U_{0: N}\right\rangle V_{l}$ for $\mathrm{GC}$ and $C_{N l}=R_{l} U_{0: N} \rho_{0}+\kappa U_{0: N}$ for SC. The revised update rule is

$$
\begin{aligned}
u_{j k}^{(i)}= & (1-\delta) \tilde{u}_{j k}^{(i-1)}+\frac{\delta}{\lambda_{k}} \operatorname{Im}\left\langle B_{j}^{(i-1)} \mid A_{k} U_{0: j-1}^{(i)}\right\rangle \\
& +\frac{\alpha \delta}{L} \sum_{l=1}^{L}\left[\tilde{v}_{j k l}-\frac{1}{\lambda_{k}}\left\langle C_{j l}^{(i-1)} \mid A_{k} U_{0: j-1}^{(i)}\right\rangle\right],
\end{aligned}
$$

where $\tilde{v}_{j k l}^{(i)}=\frac{\alpha \eta}{L}\left[u_{j k}^{(i)}-\frac{1}{\lambda_{k}} \sum_{l=1}^{L} \operatorname{Im}\left\langle C_{j l}^{(i)} \mid A_{k} U_{0: j}^{(i)}\right\rangle\right]$ and $\alpha$ is the push weight as in Eq. (2).

\section{NUMERICAL ANALYSIS}

The results of PPOQC analysis in a model two-qubit Isingcoupled system are summarized in Fig. 2. For GC, we use the controlled-NOT (CNOT) gate as the target, while for SC, the task is a transfer from the $|00\rangle$ state to the singlet state $\left|S_{0}\right\rangle=(|01\rangle-|10\rangle) / \sqrt{2}$. In each case, we use a fixed set of 100 random guess sequences. The PP-GRAPE and PPKrotov algorithms are run for various sizes of orthogonal sets $(L \in[1,15]$ with push weight $\alpha=0.2)$ and compared with the pull-only $(L=0)$ results [Figs. 2(a)-2(d)]. PPOQC outperforms the pull-only algorithms in terms of the mean final fidelity in all cases [Figs. 2(e)-2(h)]. More importantly, while the pull-only fidelities tend to saturate by settling into local minima, the push-pull trials appear to explore larger parameter space and thereby extract solutions with better fidelities. While the computational time for PP-GRAPE is 

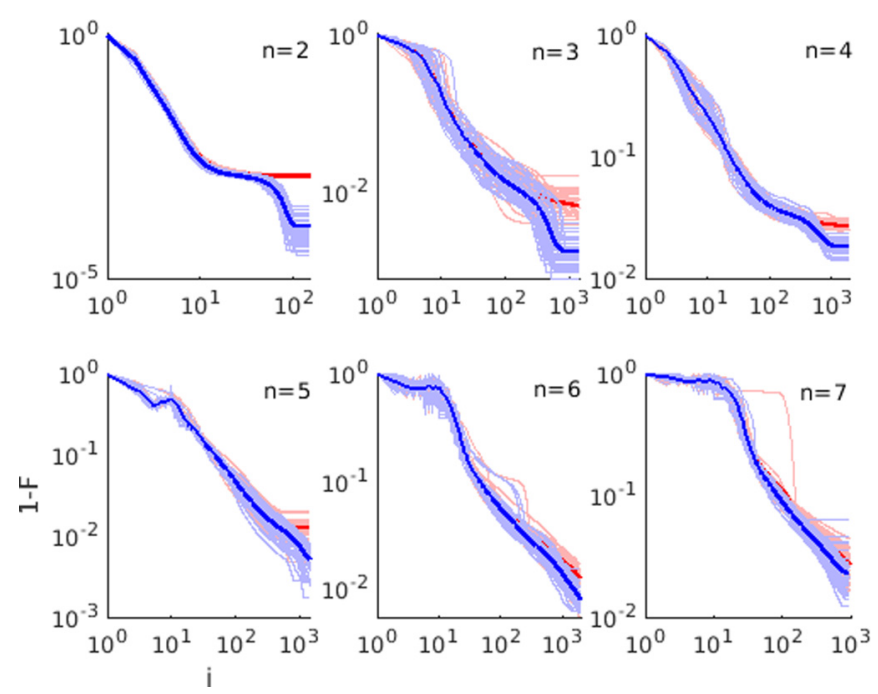

FIG. 3. Infidelities for 40 random guesses (thin lines) and their mean (thick lines) versus iteration number $i$ with Krotov (red) and with PP-Krotov (blue) ( $L=1$ and $\alpha=0.2$ ) for QFT on qubit registers of varying sizes ( $n$ as indicated).

weakly dependent on $L$, we find a slow but linear increase in the case of PP-Krotov [Figs. 2(i)-2(1)]. To quantify the advantage of PPOQC over the standard algorithms, we define the advantage factor $[1-F(L=0)] /\left[1-F\left(L_{\text {best }}\right)\right]$, where $L_{\text {best }}$ corresponds to the one with maximum mean of final fidelity [Figs. 2(m)-2(p)]. In all cases PPOQC $(L \geqslant 1)$ results in convergences superior to the standard pull-only $(L=0)$ algorithms. In particular, PP-Grape SC and PP-Krotov GC reach advantage factors up to 64, while PP-Krotov SC reaches up to 16. Only in PP-Grape GC, is the advantage factor a modest 2 .

To analyze the performance of PPOQC in larger systems, we implement a quantum Fourier transform (QFT), which is central to several important quantum algorithms [9]. We implement the entire $n$-qubit QFT circuit, consisting of $n$ local and $O\left(n^{2}\right)$ conditional gates, into a single PP-Krotov GC sequence. The results, with registers up to seven qubits, shown in Fig. 3 ensure that the PPOQC advantage persists even in larger systems.

The push-pull technique can also be combined with other convergence improvement techniques such as a conjugate gradient [35], which is illustrated by the best performance of the conjugate PP-GRAPE in Fig. 4(a). Moreover, in the pull-only algorithms, the step size $\epsilon$ may be optimized to ensure the best convergence. Similarly, in PPOQC, one can simultaneously optimize both the step size $\epsilon$ and the push weight $\alpha$ to realize the best convergence rate. This is illustrated in Fig. 4(b). Notice that we now obtain an order of magnitude improvement in the infidelity compared to the pullonly algorithm. Further discussion and numerical analysis are provided in the Appendixes.

\section{NMR EXPERIMENTS}

We now study the efficacy of PPOQC via an important application in NMR spectroscopy, i.e., preparation of a longlived state (LLS). Carravetta et al. demonstrated that the
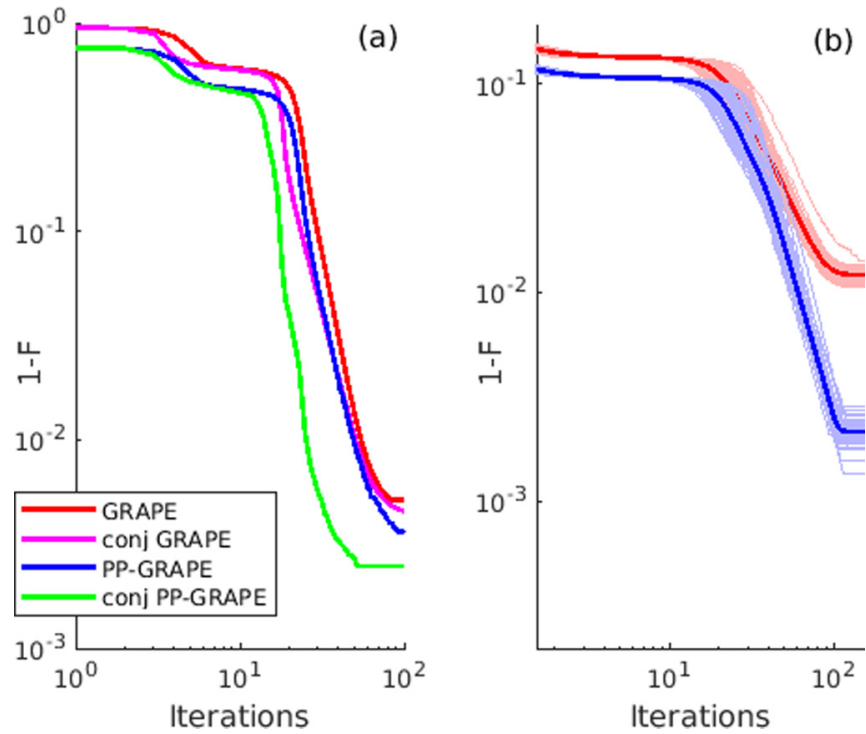

FIG. 4. Mean infidelities (thick lines) of GRAPE sequences implementing a two-qubit CNOT gate. (a) Performance with/without conjugate gradients. (b) Pull-only method $(L=0)$ with adoptive step size $\epsilon$ (red) and push-pull method $(L=15)$ with simultaneously adopted step size and push weight $\alpha$ (blue).

singlet order of a homonuclear spin pair outlives the usual lifetimes imposed by a spin-lattice relaxation time constant $\left(T_{1}\right)$ [36,37]. Prompted by numerous applications in spectroscopy and imaging, several efficient ways of preparing an LLS have been explored [38]. In the following, we utilize PP-Krotov SC optimization for this purpose.

We prepare an LLS on two protons of 2,3,6-trichlorophenol (TCP) [see Fig. 5(a)]. The sample consists of $7 \mathrm{mg}$ of TCP dissolved in $0.6 \mathrm{ml}$ of deuterated dimethyl sulfoxide. The experiments are carried out on a Bruker 500-MHz NMR spectrometer at an ambient temperature of $300 \mathrm{~K}$. The standard NMR spectrum of TCP shown in Fig. 5(a) indicates resonance offset frequencies $\pm \Delta v / 2$ to be $\pm 63.8 \mathrm{~Hz}$ and the scalar coupling constant $\mathcal{J}=8.8 \mathrm{~Hz}$. The internal Hamiltonian of the system, in a frame rotating about the direction of the Zeeman field at an average Larmor frequency, is $H_{0}=-\pi \Delta \nu I_{z}^{A}+$ $\pi \Delta v I_{z}^{B}+2 \pi \mathcal{J} I_{z}^{A} I_{z}^{B}$, where $I_{z}^{A}$ and $I_{z}^{B}$ are the $z$ components of the spin angular momentum operators $\mathbf{I}^{A}$ and $\mathbf{I}^{B}$, respectively.

The thermal equilibrium state at the high-field and hightemperature approximation is of the form $\rho_{0}=I_{z}^{A}+I_{z}^{B}$ (up to an identity term representing the background population). The goal is to design an rf sequence $\left\{u_{x}(t), u_{y}(t)\right\}$ introducing a time-dependent Hamiltonian

$$
H(t)=H_{0}+u_{x}(t)\left(I_{x}^{A}+I_{x}^{B}\right)+u_{y}(t)\left(I_{y}^{A}+I_{y}^{B}\right)
$$

that efficiently transfers $\rho_{0}$ into zero-quantum singlet-triplet order $\rho_{S}=-\mathbf{I}^{A} \cdot \mathbf{I}^{B}$. Under an rf spin lock the triplet order decays rapidly while the singlet order $\rho_{\mathrm{LLS}}$ remains long lived. The PP-Krotov SC pulse sequence shown in Fig. 5(b) consists of 1000 segments in a total duration of $45 \mathrm{~ms}$, which is $30 \%$ shorter than the standard sequence that requires $\frac{1}{2 \mathcal{J}}+\frac{3}{4 \Delta v}=$ $63 \mathrm{~ms}$ [37]. The fidelity profile shown in Fig. 5(c) indicates the robustness of the sequence against a $10 \%$ rf inhomogeneity 

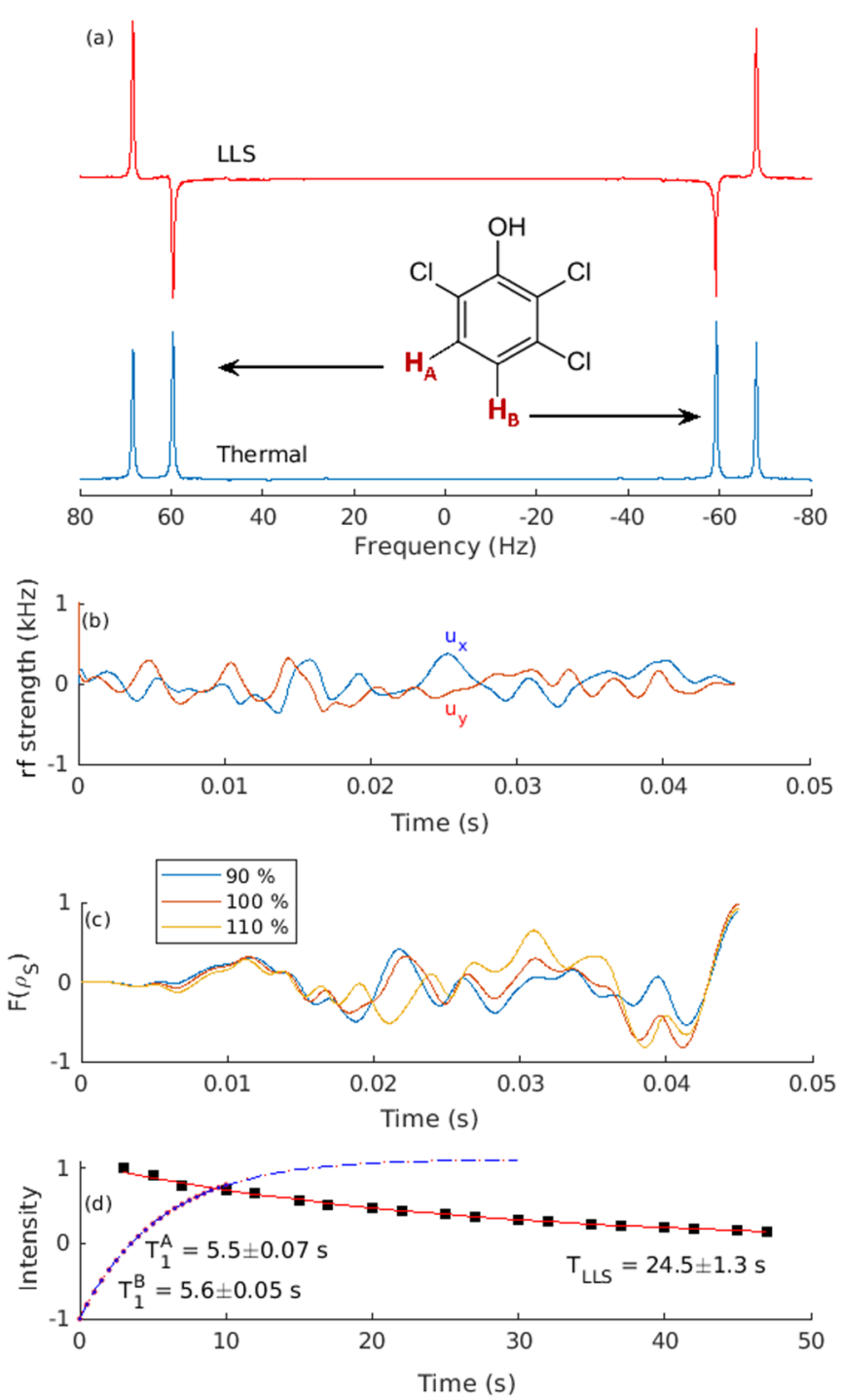

FIG. 5. (a) Thermal and LLS spectra of TCP (molecule in inset). (b) PP-Krotov SC sequence $(L=5)$ preparing LLS directly from the thermal state. (c) LLS fidelity evolution during the sequence in (b) at different rf inhomogeneity levels. (d) The $T_{1}$ values measured by the inversion recovery experiment and the $T_{\mathrm{LLS}}$ measured by storage under spin lock.

distribution with an average final fidelity above $95 \%$. The LLS spectrum shown in Fig. 5(a) is characteristic of the singlet state $\rho_{S}$. Figure 5(d) shows the experimental results of LLS storage under $1-\mathrm{kHz}$ WALTZ-16 spin lock. It confirms the long lifetime $T_{\mathrm{LLS}}$ of about $24.5 \mathrm{~s}$ or about 4.5 times $T_{1}^{A}$ and $T_{1}^{B}$ measured by inversion recovery experiments. A comparison with the standard method (as in Ref. [37]) revealed 27\% higher singlet order, further indicating the superiority of the PP-Krotov SC sequence.

\section{CONCLUSION}

At the heart of optimization algorithms lies a performance function that evaluates a process in relation to a target. Using a hybrid objective function that simultaneously takes into account a given target operator and a set of orthogonal operators, we devised the push-pull optimization of quantum controls. The combined influences of these operators not only results in a faster convergence of the optimization algorithm, but also effects a better exploration of the parameter space and thereby generates better solutions. Although the orthogonal set grows exponentially with the system size, it is not necessary to include an exhaustive set. Even a small set of orthogonal operators, generated randomly during the iterations, can bring about a significant improvement in convergence.

While the push-pull approach can be implemented in a wide variety of quantum control routines, we described adopting it in gradient-based and variational-principle-based optimizations. We observed considerable improvements in the convergence rates, without overburdening computational costs. The numerical analysis with up to seven qubits confirmed that the push-pull method retained superiority even in larger systems. Combining the push-pull method with conjugate gradients also resulted in better performance. Numerical analysis revealed further improvement with the adoptive step sizes and adoptive push weights.

Finally, using NMR methods, we experimentally verified the robustness of a push-pull Krotov control sequence preparing a long-lived singlet order. Further work in this direction should include optimizing the functional forms of orthogonal gradients and generalization to open quantum controls.

\section{ACKNOWLEDGMENTS}

Discussions with Sudheer Kumar, Deepak Khurana, Soham Pal, Gaurav Bhole, Hemant Katiyar, and Pranay Goel are

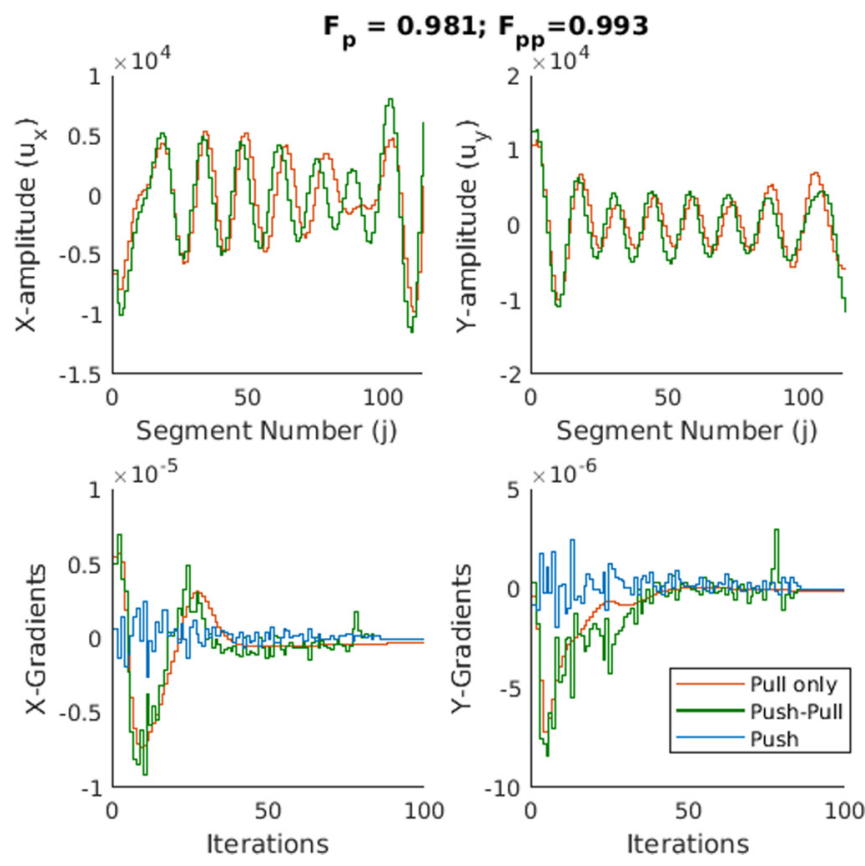

FIG. 6. Shown on top are the $X$ and $Y$ amplitudes for a two-qubit CNOT gate with pull-only GRAPE (red) and push-pull PP-GRAPE (green) $(L=5)$ algorithms. Shown on the bottom are the evolutions of the $X$ and $Y$ gradients versus iteration number for one particular segment (segment number 78). Notice how the mean push gradients (blue) from the orthogonal operators modulate the effective push-pull gradients (green). 
gratefully acknowledged. This work was partly supported by DST/SJF/PSA-03/2012-13 and CSIR 03(1345)/16/EMR-II.

\section{APPENDIX A: RAPID PARAMETER SEARCH IN THE PUSH-PULL APPROACH}

To gain insight into the superiority of the push-pull approach over the pull-only approach, we observed how the gradients evolve over time. Figure 6 displays the evolution of gradients versus control amplitudes over several iterations. The simulations are carried out for a two-qubit CNOT gate with both pull-only and push-pull GRAPE algorithms. The push-pull algorithm ultimately converged to a better fidelity (0.993) than the pull-only algorithm (0.981). Notice that the push-pull gradients show more rapid changes than the pullonly algorithm, indicating a more robust parameter search in action. This behavior appears to be the crucial factor for the faster convergence of the push-pull approach.

\section{APPENDIX B: PUSH WEIGHT}

Figure 7 displays infidelities of PP-GRAPE and PP-Krotov algorithms versus the push $\alpha$. We note that, on the positive side, the infidelity is generally superior to the pullonly algorithm $(\alpha=0)$. In each case, there exists an optimal push weight roughly in the range $\alpha \in[0.1,0.3]$ at which the
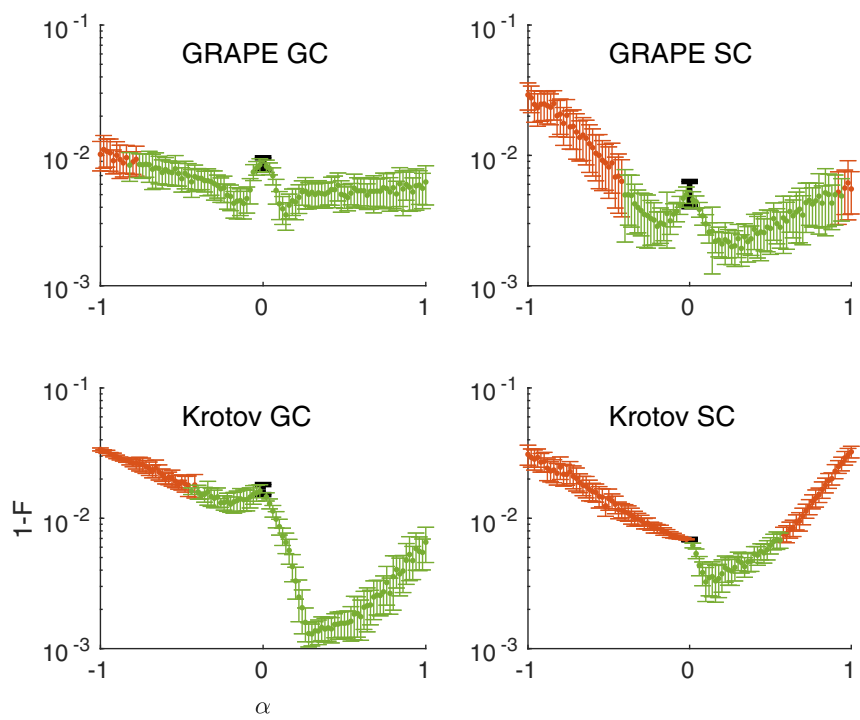

FIG. 7. Infidelity versus the push weight $\alpha$ for $L=6$. Error bars indicate one standard deviation. The black point at $\alpha=0$ corresponds to the standard pull-only algorithms. The green and red regions indicate, respectively, superior and inferior performances of PPOQC with respect to the pull-only algorithm.

PPOQC works best. It is interesting to see that some negative regions also display superior performances.
[1] A. E. Bryson, Jr. and Y.-C. Ho, Applied Optimal Control: Optimization, Estimation, and Control (Routledge, New York, 2018).

[2] L. S. Pontryagin, Mathematical Theory of Optimal Processes (Routledge, New York, 2018).

[3] J. Werschnik and E. Gross, J. Phys. B 40, R175 (2007).

[4] D. Dong and I. R. Petersen, IET Control Theory Appl. 4, 2651 (2010).

[5] P. Brumer and M. Shapiro, Acc. Chem. Res. 22, 407 (1989).

[6] L. Petzold and W. Zhu, AIChE J. 45, 869 (1999).

[7] D. J. Tannor and S. A. Rice, J. Chem. Phys. 83, 5013 (1985).

[8] W. Zhu, J. Botina, and H. Rabitz, J. Chem. Phys. 108, 1953 (1998).

[9] N. C. Nielsen, C. Kehlet, S. J. Glaser, and N. Khaneja, eMagRes (2010), doi: 10.1002/9780470034590.emrstm1043.

[10] J. P. Palao and R. Kosloff, Phys. Rev. Lett. 89, 188301 (2002).

[11] P. Doria, T. Calarco, and S. Montangero, Phys. Rev. Lett. 106, 190501 (2011).

[12] S. J. Glaser et al., Eur. Phys. J. D 69, 279 (2015).

[13] E. M. Fortunato, M. A. Pravia, N. Boulant, G. Teklemariam, T. F. Havel, and D. G. Cory, J. Chem. Phys. 116, 7599 (2002).

[14] N. Khaneja, T. Reiss, C. Kehlet, T. Schulte-Herbrüggen, and S. J. Glaser, J. Magn. Reson. 172, 296 (2005).

[15] P. De Fouquieres, S. Schirmer, S. Glaser, and I. Kuprov, J. Magn. Reson. 212, 412 (2011)

[16] S. Machnes, E. Assémat, D. Tannor, and F. K. Wilhelm, Phys. Rev. Lett. 120, 150401 (2018).

[17] V. F. Krotov, Dokl. Math. 78, 949 (2008).
[18] I. I. Maximov, Z. Tošner, and N. C. Nielsen, J. Chem. Phys. 128, 184505 (2008).

[19] D. M. Reich, M. Ndong, and C. P. Koch, J. Chem. Phys. 136, 104103 (2012).

[20] T. Caneva, T. Calarco, and S. Montangero, Phys. Rev. A 84, 022326 (2011).

[21] J. J. W. H. Sørensen, M. O. Aranburu, T. Heinzel, and J. F. Sherson, Phys. Rev. A 98, 022119 (2018).

[22] G. Bhole, V. S. Anjusha, and T. S. Mahesh, Phys. Rev. A 93, 042339 (2016).

[23] D. Khurana and T. S. Mahesh, J. Magn. Reson. 284, 8 (2017).

[24] C. Chen, D. Dong, H.-X. Li, J. Chu, and T.-J. Tarn, IEEE Trans. Neural Networks Learn. Syst. 25, 920 (2013).

[25] X.-M. Zhang, Z. Wei, R. Asad, X.-C. Yang, and X. Wang, npj Quantum Inf. 5, 85 (2019).

[26] L. M. Vandersypen and I. L. Chuang, Rev. Mod. Phys. 76, 1037 (2005).

[27] F. Dolde, V. Bergholm, Y. Wang, I. Jakobi, B. Naydenov, S. Pezzagna, J. Meijer, F. Jelezko, P. Neumann, T. SchulteHerbrüggen et al., Nat. Commun. 5, 3371 (2014).

[28] K. Singer, U. Poschinger, M. Murphy, P. Ivanov, F. Ziesel, T. Calarco, and F. Schmidt-Kaler, Rev. Mod. Phys. 82, 2609 (2010).

[29] Y.-P. Shim and C. Tahan, Nat. Commun. 7, 11059 (2016).

[30] M. S. Vinding, I. I. Maximov, Z. Tošner, and N. C. Nielsen, J. Chem. Phys. 137, 054203 (2012).

[31] R. Chakrabarti and H. Rabitz, Int. Rev. Phys. Chem. 26, 671 (2007).

[32] A. N. Pechen and D. J. Tannor, Phys. Rev. Lett. 106, 120402 (2011). 
[33] R. Shankar, Principles of Quantum Mechanics (Springer Science + Business Media, New York, 2012).

[34] V. Krotov, Global Methods in Optimal Control Theory (CRC, Boca Raton, 1995), Vol. 195.

[35] J. R. Shewchuk, An introduction to the conjugate gradient method without the agonizing pain, Carnegie Mellon University report, 1994 (unpublished).
[36] M. Carravetta, O. G. Johannessen, and M. H. Levitt, Phys. Rev. Lett. 92, 153003 (2004).

[37] M. Carravetta and M. H. Levitt, J. Am. Chem. Soc. 126, 6228 (2004).

[38] G. Pileio, Prog. Nucl. Magn. Reson. Spectrosc. 98-99, 1 (2017). 